\title{
New Lower Bounds for the Shannon Capacity of Odd Cycles
}

\author{
K. Ashik Mathew and Patric R. J. Östergård *
}

April 8, 2015

\begin{abstract}
The Shannon capacity of a graph $G$ is defined as $c(G)=\sup _{d \geq 1}\left(\alpha\left(G^{d}\right)\right)^{\frac{1}{d}}$, where $\alpha(G)$ is the independence number of $G$. The Shannon capacity of the cycle $C_{5}$ on 5 vertices was determined by Lovász in 1979 , but the Shannon capacity of a cycle $C_{p}$ for general odd $p$ remains one of the most notorious open problems in information theory. By prescribing stabilizers for the independent sets in $C_{p}^{d}$ and using stochastic search methods, we show that $\alpha\left(C_{7}^{5}\right) \geq 350, \alpha\left(C_{11}^{4}\right) \geq 748, \alpha\left(C_{13}^{4}\right) \geq 1534$ and $\alpha\left(C_{15}^{3}\right) \geq 381$. This leads to improved lower bounds on the Shannon capacity of $C_{7}$ and $C_{15}: c\left(C_{7}\right) \geq 350^{\frac{1}{5}}>3.2271$ and $c\left(C_{15}\right) \geq 381^{\frac{1}{3}}>7.2495$.
\end{abstract}

\section{Introduction}

The Shannon capacity of a graph is an important information-theoretic parameter and plays a central role in the study of the zero-error capacity of a noisy communication channel represented by the graph [18. A communication channel transmitting $p$ different symbols can be represented by a graph $G$ with vertex set $V$ and edge set $E$ in the following way: $V$ is the set of transmitted symbols, and for $v_{1}, v_{2} \in V,\left(v_{1}, v_{2}\right) \in E$ if the symbols $v_{1}$ and $v_{2}$ are indistinguishable. The Shannon capacity of $G$ is defined as

$$
c(G)=\sup _{d \geq 1}\left(\alpha\left(G^{d}\right)\right)^{\frac{1}{d}},
$$

where $\alpha(G)$ is the independence number of $G$ and the graph strong product is assumed [24]. For a survey of some of the early results related to the Shannon capacity of graphs, see [17.

Algebraic tools for the study of Shannon capacity were proposed by Haemers [12, 13. while the Shannon capacity of digraphs were investigated by Alon [1]. See also [2, 3, 10, 26] for some related studies.

*K. A. Mathew and P. R. J. Östergård are with the Department of Communications and Networking, Aalto University School of Electrical Engineering, P.O. Box 13000, 00076 Aalto, Finland (e-mail: ashik.kizhakkepallathu@aalto.fi; patric.ostergard@aalto.fi). 
For a channel transmitting $p$ symbols represented by the elements of $\mathbb{Z}_{p}=$ $\{0,1,2, \ldots, p-1\}$ and where two distinct symbols $s$ and $t$ are indistinguishable if $s-t \equiv \pm 1(\bmod p)$, the graph that represents the channel is $C_{p}$, the cycle on $p$ vertices. If $p$ is even, then $c\left(C_{p}\right)=p / 2$. It was shown by Lovász [19] in 1979 that $c\left(C_{5}\right)=\sqrt{5}$, but finding the Shannon capacity of $C_{p}$ for $p \geq 7$ and odd is still open [5].

It is well known that the independence number of $C_{p}^{d}$, the $d$ th power (under strong product) of a cycle $C_{p}$, is same as the number of hypercubes of side 2 that can be packed in a discrete $d$-dimensional torus of width $p$, denoted by $G(d, p)$ [9]. See Figure 1 for a visualization of such a 2-dimensional packing and a corresponding independent set in $C_{5}^{2}$. Representing independent sets as a packing of cubes often gives a more comprehensible model (visually) to work with. This is especially true when we talk about symmetries, though for the cause of adhering to formalism, we stick to a rather algebraic notion to discuss symmetries in the remaining sections.

Cube packings and their different variants also form the basis of several classical and well-studied problems in combinatorics [9, 16]. The function $G(d, p)$ has been studied thoroughly, and exact values and bounds have been published in [4] and later studies. Several of these results have been obtained using exhaustive and stochastic computational methods. For example, Baumert et al. [4] used exhaustive search to show that $G(3,7)=33$, and Vesel and Žerovnik 25] proved that $G(4,7) \geq 108$ with simulated annealing. The current authors used another stochastic (local search) method, tabu search, to obtain lower bounds for the capacity of triangular graphs 20. (Triangular graphs are closely related to cycle graphs; the capacity problem for triangular graphs can be studied via a generalization of the cube packing problem.)

Many of the best known cube packings possess some kind of symmetry. For example, the packing in Figure 1 has a symmetry generated by $(a, b) \rightarrow$ $(a+2, b+1)$ (addition modulo 5). This symmetry generates a group of order 5. Several additional examples can be found in the constructions of [4].

In the current work, stochastic computational methods will be combined with the idea of prescribing symmetries of packings. By prescribing symmetries, one is able to speed up the computer search. Obviously, such a search has a possibility of success only if there are packings with the given symmetries. By exploiting possible symmetries in as exhaustive manner as possible, we are able to show that $\alpha\left(C_{7}^{5}\right) \geq 350, \alpha\left(C_{11}^{4}\right) \geq 748, \alpha\left(C_{13}^{4}\right) \geq 1534$ and $\alpha\left(C_{15}^{3}\right) \geq 381$. These bounds further imply that $c\left(C_{7}\right) \geq 350^{\frac{1}{5}}>3.2271$ and $c\left(C_{15}\right) \geq 381^{\frac{1}{3}}>$ 7.2495 .

The paper is organized as follows. In Section 2 the approach of prescribing symmetries is considered, and a stochastic local search method for finding packings is discussed in Section 3 . In Section 4, the results are summarizes and tabulated. Specific packings are listed in the Appendix. 


\begin{tabular}{|l|l|l|l|l|}
\hline & 4 & 4 & 5 & 5 \\
\hline 3 & 4 & 4 & & 3 \\
\hline 3 & & 2 & 2 & 3 \\
\hline 1 & 1 & 2 & 2 & \\
\hline 1 & 1 & & 5 & 5 \\
\hline
\end{tabular}

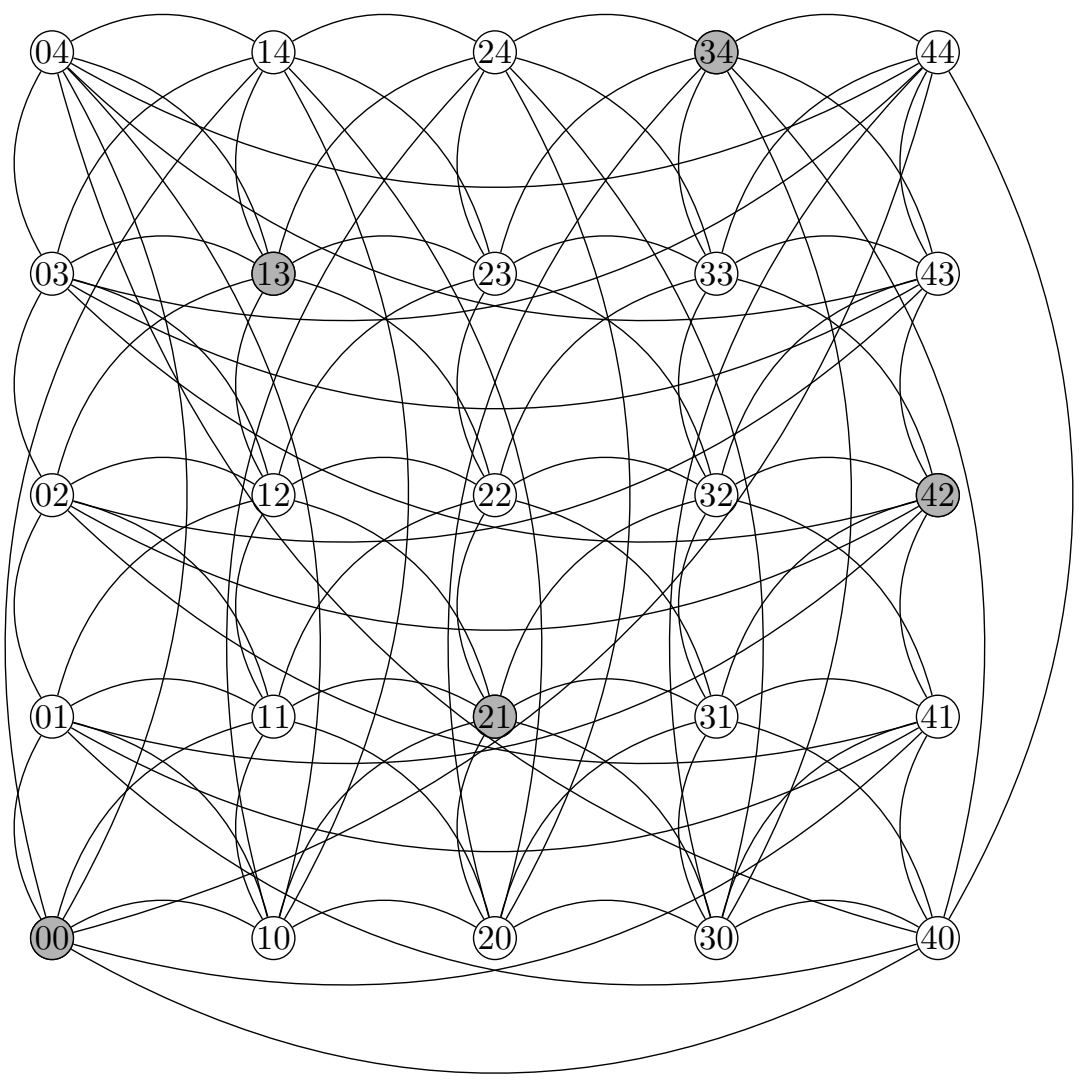

Figure 1: Packing a torus with 2-dimensional cubes and the corresponding independent set in $C_{5}^{2}$ 


\section{$2 \quad$ Prescribing Symmetries of Independent Sets}

The graph $G=C_{p}^{d}$ is conveniently discussed in the framework of codes. Let $V(G)=\{0,1, \ldots, p-1\}^{d}$, the set of all codewords of length $d$ over $\mathbb{Z}_{p}$. For $v \in V$, we denote $v=\left(v_{1}, v_{2}, \ldots, v_{d}\right)$. Now we define the set of edges as

$$
E(G)=\left\{\left\{v, v^{\prime}\right\}: v, v^{\prime} \in V(G) \text { and } \max _{1 \leq i \leq d} \min \left\{\left|v_{i}-v_{i}^{\prime}\right|, p-\left|v_{i}-v_{i}^{\prime}\right|\right\}<2\right\} .
$$

This definition further shows the one-to-one correspondence between an independent set in $C_{p}^{d}$ and a packing in the discrete $d$-dimensional torus of width $p$ by (hyper)cubes of side 2 (with their centers - or any other specific position of the cubes - in the position given by the element of the independent set).

For small parameters, one may find the independence number of $C_{p}^{d}$ using Cliquer 22] or some other available software. However, growing parameters makes the use of such exact algorithms infeasible at some point. One way of handling large instances of combinatorial search problems is to prescribe symmetries [15, Chapter 9].

Symmetries of an independent set in a graph $G$ are elements of the automorphism group of $G$ - denoted by $\operatorname{Aut}(G)$ - that stabilize the independent set.

Let $N[v]$ denote the closed neighborhood of the vertex $v$. A graph $G$ is called thin if $N[u] \neq N[v]$ whenever $u \neq v$. A prime graph $G$ is one that cannot be written as $G=G_{1} \otimes G_{2}$ (strong product of $G_{1}$ and $G_{2}$ ) for non-trivial graphs $G_{1}$ and $G_{2}$.

Theorem 2.1 ([14, Theorem 7.18). For a graph $G=G_{1} \otimes G_{2} \otimes \cdots \otimes G_{n}$ where $G_{1}, G_{2}, \ldots, G_{n}$ are connected, thin and prime graphs, the automorphism group of $G$ is isomorphic to the automorphism group of the disjoint union of graphs $G_{1}, G_{2}, \ldots, G_{n}$.

Theorem 2.2 (Frucht [11). If $G$ is a connected graph and $n G$ denotes the graph representing $n$ disjoint copies of $G$, then $\operatorname{Aut}(n G)$ is the wreath product $\operatorname{Aut}(G) \curlywedge S_{n}$.

Theorem 2.3. The automorphism group of $C_{p}^{d}$ (for $p>3$ ) is isomorphic to the wreath product $D_{p} \prec S_{d}$, where $D_{p}$ is the dihedral group of order $2 p$ and $S_{d}$ is the symmetric group of degree $d$.

Proof. We first show that $C_{p}$ on vertices $\{0,1,2, \ldots, p-1\}$ and edge set $\{\{u, v\}$ : $u-v= \pm 1 \bmod p\}$ is thin and prime for $p>3$. Consider any two distinct vertices $x, y \in V\left(C_{p}\right)$. If $\{x, y\} \notin E\left(C_{p}\right), x \notin N[y]$ and so $N[x] \neq N[y]$. Suppose $\{x, y\} \in E\left(C_{p}\right)$. This means that (w.l.o.g.) $x-y=1 \bmod p$. Consider the vertex $z=x+1 \bmod p$. Clearly, $\{x, z\} \in E\left(C_{p}\right)$ and $\{y, z\} \notin E\left(C_{p}\right)$, leading to $N[x] \neq N[y]$. So, $C_{p}$ is thin. Now we observe that $C_{p}$ is prime by the following argument. By definition, if $G=G_{1} \otimes G_{2}$ is connected, both $G_{1}$ and $G_{2}$ are connected. Since $K_{2} \otimes K_{2}=K_{4}$, the strong product of any two graphs with at least one edge each has $K_{4}$ as a subgraph. Since $C_{p}$ does not have $K_{4}$ as a subgraph, $C_{p}$ is prime. Now that $C_{p}$ is connected, thin and prime, Theorem 2.1 applies to it. Since the automorphism group of $C_{p}$ is the dihedral group $D_{p}$, using Theorem 2.2, the result follows. 
The order of the group $\operatorname{Aut}\left(C_{p}^{d}\right)$ is $\left|\operatorname{Aut}\left(C_{p}^{d}\right)\right|=\left|D_{p} \curlywedge S_{d}\right|=(2 p)^{d} d$ !. In the framework of codes, introduced earlier, elements of $\operatorname{Aut}\left(C_{p}^{d}\right)$ act by a permutation of the coordinates followed by permutations of the coordinate values (separately for each coordinate) that have the form

$$
i \rightarrow a i+b(\bmod p), a \in\{-1,1\}, b \in \mathbb{Z}_{p},
$$

Given two codes corresponding to independent sets or packings of cubes, we say that these are equivalent if one of the codes can be obtained from the other with a mapping in the action of $\operatorname{Aut}\left(C_{p}^{d}\right)$. Such mappings from a code onto itself - which are formally stabilizers of the code (and the corresponding independent set and the packing) under the action of $\operatorname{Aut}\left(C_{p}^{d}\right)$ - are said to form the automorphism group of the code.

The automorphism group of a code is a subgroup of $\operatorname{Aut}\left(C_{p}^{d}\right)$. When prescribing possible automorphism groups, we therefore consider subgroups of $\operatorname{Aut}\left(C_{p}^{d}\right)$ up to conjugacy. Moreover, we reduce the number of groups to consider by explicitly restricting the computations to cyclic groups. (In this manner we are still able to cover a large part of the groups, since most large groups that are omitted will have a cyclic subgroup amongst the groups considered.)

Having prescribed an automorphism group of a code, the action of the group partitions all possible codewords into orbits. In the framework of independent sets, we now get instances of the maximum weight independent set problem. The vertex set consist of all admissible orbits: the pairs of codewords in the set must fulfill the distance criterion in (1). The weight of a vertex is the number of codewords in the orbit. Finally, edges are inserted whenever no pairs of codewords, one from each of the orbits, violate the distance criterion in (1).

With prescribed automorphism groups, we can extend the range of parameters for which the running time of Cliquer (which can also handle weighted graphs) or similar software is feasibly short. Moreover, by also changing the computational approach from being exact to becoming stochastic, we can extend the range of parameters even further. Such an approach will be discussed next.

\section{Stochastic Search for Weighted Independent Sets}

The graphs obtained in the previous section are weighted. In general, let $G$ be an arbitrary graph with vertex set $V(G)$ and edge set $E(G)$, where each vertex has a positive integer weight. This is obviously a generalization of the case of maximum independent sets, which we get by letting all weights be 1 . Note that since an independent graph corresponds to a clique in the complement graph, any discussion of independent sets apply to cliques and vice versa. The maximum (weight) independent set problem is surveyed in [7, in the framework of cliques. 
The decision problem of finding an independent set of weight at least $k$ in a graph $G$ is NP-complete, so no polynomial-time general algorithms are expected to be discovered. Due to the hardness (and the importance) of the problem, a lot of effort has been put on developing stochastic algorithms. For unweighted graphs and with stochastic algorithms, the main approach has been to process an independent set by adding and removing vertices. Unfortunately, such a straightforward approach is not as effective when the vertices have different weights.

Montemanni and Smith 21] discovered a technique for modifying independent sets (in terms of cliques) by removing not one but many vertices at a time. After removing a set of vertices, an exact algorithm (like Cliquer) can be used to find a set of vertices to add that have the largest possible weight. In some sense, this approach lies in between basic stochastic search and exact algorithms.

The main decision to be made in the approach by Montemanni and Smith is the set of vertices to remove from an independent set. In 21 vertices are removed in a random manner. When one thinks about this problem in the context of cube packings, removal means removing (hyper)cubes. When cubes are removed, there will be holes in the packing. But with such holes that are not connected, we will have a situation equivalent to that of sequentially removing a smaller number of cubes in different parts of the packing.

The second author [23] realized that with instances of the maximum weight independent set problem that come from packing problems, one may remove one vertex $v$ and all vertices that are within a certain heuristic distance from $v$. The heuristic distance, which does not have to be a metric, is defined separately for each pair of vertices of a graph, and some experimenting is typically needed to find a proper definition. The approach in [23] has been used to find new $q$-analog packings 8 .

We here use the algorithm developed in [23] and define the distance between vertices of the weighted graphs as the minimum of

$$
d\left(v, v^{\prime}\right)=\sum_{i=1}^{d} \min \left\{\left|v_{i}-v_{i}^{\prime}\right|, p-\left|v_{i}-v_{i}^{\prime}\right|\right\} .
$$

over all pairs of codewords, with one codeword from each orbit.

\section{Results}

By applying the approaches discussed in this paper and using more than 2 CPUyears in the stochastic search, we have obtained independent sets that attain the following bounds for $G(d, p): G(5,7) \geq 350, G(4,11) \geq 748, G(4,13) \geq 1534$ and $G(3,15) \geq 381$. These also leads to improved lower bounds on the Shannon capacity of $C_{7}$ and $C_{15}: c\left(C_{7}\right) \geq 350^{\frac{1}{5}}>3.2271$ and $c\left(C_{15}\right) \geq 381^{\frac{1}{3}}>7.2495$. There previous best known lower bounds for $c\left(C_{7}\right)$ and $c\left(C_{15}\right)$ were $108^{\frac{1}{4}}>$ 3.2237 [25] and $380^{\frac{1}{3}}>7.2431$ [4] respectively. The best known lower bounds for $c\left(C_{p}\right)$ for other small cycles of odd length are: $c\left(C_{9}\right) \geq 81^{\frac{1}{3}}>4.3267$ [4], 
$c\left(C_{11}\right) \geq 148^{\frac{1}{3}}>5.2895$ [4 and $c\left(C_{13}\right) \geq 247^{\frac{1}{3}}>6.2743$ [6]. The Lovász's $\vartheta$-function

$$
\vartheta(p)=\frac{p \cos \frac{\pi}{p}}{1+\cos \frac{\pi}{p}}
$$

gives upper bounds for the Shannon capacity of the odd cycle $C_{p}$ [19]. Using this function, $c\left(C_{7}\right)<3.3177, c\left(C_{9}\right)<4.3601, c\left(C_{11}\right)<5.3864, c\left(C_{13}\right)<6.4042$ and $c\left(C_{15}\right)<7.4172$.

The currently best known upper and lower bounds for $G(d, p)$ are listed in Table 1 together with keys. Only one key is provided in cases where the value can be obtained using more than one method.

Table 1: Bounds on $G(d, p)$

\begin{tabular}{cccccc}
\hline$p \backslash d$ & 1 & 2 & 3 & 4 & 5 \\
\hline 5 & ${ }^{a} 2^{a}$ & ${ }^{a} 5^{a}$ & ${ }^{c} 10^{f}$ & ${ }^{c} 25^{d}$ & ${ }^{c} 50-55^{j}$ \\
7 & ${ }^{a} 3^{a}$ & ${ }^{a} 10^{a}$ & ${ }^{f} 33^{f}$ & ${ }^{h} 108-115^{d}$ & ${ }^{k} 350-401^{j}$ \\
9 & ${ }^{a} 4^{a}$ & ${ }^{a} 18^{a}$ & ${ }^{e} 81^{d}$ & ${ }^{c} 324-361^{j}$ & ${ }^{c} 1458-1575^{j}$ \\
11 & ${ }^{a} 5^{a}$ & ${ }^{a} 27^{a}$ & ${ }^{e} 148^{d}$ & ${ }^{k} 748-814^{d}$ & ${ }^{c} 3996-4477^{d}$ \\
13 & ${ }^{a} 6^{a}$ & ${ }^{a} 39^{a}$ & ${ }^{g} 247^{i}$ & ${ }^{k} 1534-1605^{d}$ & ${ }^{c} 9633-10432^{d}$ \\
15 & ${ }^{a} 7^{a}$ & ${ }^{a} 52^{a}$ & ${ }^{k} 381-390^{d}$ & ${ }^{b} 2720-2925^{d}$ & ${ }^{c} 19812-21937^{d}$ \\
\hline
\end{tabular}

Key to Table 1

Bounds:

$$
\begin{array}{ll}
a & G(1, p)=\left\lfloor\frac{p}{2}\right\rfloor, G(2, p)=\left\lfloor\frac{p^{2}-p}{4}\right\rfloor \text { [4, Theorem 2] } \\
b & G(d, p) \geq 1+G(d, p-2) \frac{p^{d}-2^{d}}{(p-2)^{d}} \text { [4, Corollary 2] } \\
c & G(d, p) \geq G\left(d_{1}, p\right) G\left(d-d_{1}, p\right) \text { [4, Corollary 3] } \\
d & G(d, p) \leq\left\lfloor\frac{p}{2} G(d-1, p)\right\rfloor[4, \text { Lemma 2] } \\
e & \text { Baumert et al. [4, Theorem 3] } \\
f & \text { Baumert et al. [4, Theorem 4] } \\
g & \text { Baumert et al. [4, Theorem 6] } \\
h & \text { Vesel and Žerovnik [25] } \\
i & \text { Bohman, Holzman, and Natarajan [6] } \\
j & G(d, p) \leq\left[\frac{p \cos \frac{\pi}{p}}{1+\cos \frac{\pi}{p}}\right]^{d}[19] \\
k & \text { This paper, see Appendix }
\end{array}
$$

\section{Appendix}

We here list codes giving the four new lower bounds. The permutation of coordinates is the identity permutation in all generators of the groups, and $a=1$ for all value permutations in (2). We therefore present the groups by simply listing the values of $b$ for the $d$ value permutations of a generator. 


\section{$G(5,7) \geq 350$ :}

Generator: $(0,1,1,5,1)$

Group order: 7

Orbit representatives: $(0,5,6,6,0),(0,0,6,6,0),(3,3,0,6,0),(0,5,2,1,0),(2,5$, $6,5,0),(1,3,0,0,0),(2,3,2,0,0),(2,1,0,4,0),(2,5,2,1,0),(0,2,1,2,0),(4,2$, $6,3,0),(4,0,0,3,0),(5,1,2,3,0),(3,6,1,5,0),(4,5,0,2,0),(3,4,5,2,0),(1,6$, $1,6,0),(2,3,6,4,0),(5,5,1,4,0),(5,3,1,3,0),(6,4,0,1,0),(0,0,2,2,0),(6,0$, $1,0,0),(5,1,5,0,0),(5,6,6,0,0),(5,3,5,1,0),(0,3,6,5,0),(2,0,2,1,0),(4,0$, $3,5,0),(4,4,2,6,0),(4,2,3,6,0),(1,5,5,3,0),(6,2,4,5,0),(4,4,4,6,0),(6,2$, $0,0,0),(1,0,5,4,0),(4,6,4,0,0),(3,1,4,1,0),(3,6,5,2,0),(6,0,5,4,0),(2,3$, $3,2,0),(1,1,4,2,0),(1,5,3,3,0),(1,2,4,4,0),(1,1,1,6,0),(3,1,1,6,0),(0,3$, $3,2,0),(6,4,3,4,0),(6,6,3,4,0),(6,5,5,4,0)$

$G(4,11) \geq 748$ :

Generator: $(1,5,8,9)$

Group order: 11

Orbit representatives: $(9,10,0,0),(7,10,9,0),(5,4,0,0),(5,4,2,0),(2,3,0,0)$, $(7,4,2,0),(7,8,1,0),(1,10,2,0),(2,7,4,0),(0,7,5,0),(6,9,7,0),(6,6,1,0)$, $(8,6,1,0),(8,8,10,0),(4,2,1,0),(5,2,3,0),(6,4,4,0),(5,2,5,0),(3,7,6,0)$, $(2,9,6,0),(4,9,6,0),(1,9,4,0),(1,7,7,0),(5,7,8,0),(6,6,10,0),(3,2,3,0)$, $(8,4,4,0),(3,7,8,0),(10,10,2,0),(0,5,5,0),(9,6,5,0),(4,9,8,0),(4,7,10,0)$, $(0,10,0,0),(7,2,4,0),(7,2,6,0),(7,0,7,0),(6,8,10,0),(0,3,10,0),(8,6,3,0)$, $(10,6,3,0),(3,5,0,0),(1,1,1,0),(0,5,7,0),(2,5,7,0),(2,3,9,0),(1,5,9,0)$, $(3,5,9,0),(3,0,1,0),(4,0,3,0),(2,0,4,0),(3,9,4,0),(4,0,5,0),(6,0,5,0),(9$, $8,1,0),(10,1,8,0),(8,1,9,0),(1,1,10,0),(10,1,10,0),(0,8,2,0),(9,8,3,0)$, $(9,1,6,0),(10,3,6,0),(8,4,6,0),(5,0,7,0),(1,3,7,0),(10,3,8,0),(9,10,9,0)$

$G(4,13) \geq 1534:$

Generator: $(0,1,0,2)$

Group order: 13

Orbit representatives: $(9,6,7,0),(9,8,9,0),(7,1,8,0),(0,7,6,0),(8,10,8,0)$, $(7,11,0,0),(4,11,11,0),(5,4,2,0),(8,12,9,0),(3,7,10,0),(5,7,10,0),(5,0$, $1,0),(2,0,12,0),(1,7,8,0),(1,7,10,0),(0,7,4,0),(12,0,9,0),(5,2,2,0),(11$, $2,7,0),(11,0,5,0),(10,4,5,0),(1,5,5,0),(3,8,2,0),(4,0,12,0),(11,0,7,0)$, $(5,12,5,0),(3,5,7,0),(5,12,7,0),(7,12,7,0),(8,12,11,0),(2,12,3,0),(2,3$, $4,0),(4,10,4,0),(7,2,1,0),(0,9,5,0),(0,9,7,0),(1,5,7,0),(10,4,7,0),(1,1$, $2,0),(6,10,4,0),(6,10,6,0),(12,11,5,0),(12,11,7,0),(11,2,9,0),(11,4,9,0)$, $(6,3,8,0),(6,5,9,0),(7,1,10,0),(7,3,10,0),(5,9,10,0),(9,10,10,0),(6,5,11$, $0),(10,1,3,0),(4,5,9,0),(0,11,9,0),(2,11,11,0),(7,4,2,0),(4,6,3,0),(6,6$, $3,0),(9,12,3,0),(0,0,0,0),(8,1,12,0),(6,7,12,0),(6,9,12,0),(9,10,12,0)$, $(8,8,3,0),(6,8,4,0),(8,10,4,0),(11,2,5,0),(7,6,5,0),(9,6,5,0),(9,8,5,0)$, $(7,8,6,0),(8,10,6,0),(9,8,7,0),(12,1,2,0),(1,3,2,0),(0,12,2,0),(3,9,11$, $0),(2,3,7,0),(4,3,8,0),(2,9,9,0),(2,11,9,0),(7,0,1,0),(9,1,1,0),(9,12,1$, $0),(4,8,4,0),(5,1,8,0),(11,0,0,0),(11,11,0,0),(9,10,1,0),(11,12,2,0),(10$, $3,3,0),(12,10,3,0),(0,2,0,0),(11,2,0,0),(0,9,9,0),(12,2,11,0),(11,4,11$, $0),(1,9,11,0),(0,11,11,0),(2,2,0,0),(4,2,0,0),(2,4,0,0),(4,4,0,0),(0,11$, $0,0),(3,6,1,0),(3,10,2,0),(2,1,4,0),(3,12,5,0),(2,1,6,0),(5,1,6,0),(4,3$, 
$6,0),(3,7,8,0),(10,6,9,0),(12,0,11,0),(10,6,11,0),(10,8,11,0)$

$G(3,15) \geq 381:$

Generator: $(5,0,10)$

Group order: 3

Orbit representatives: $(1,10,4),(1,11,0),(10,10,0),(1,2,2),(13,3,2),(9,11,2)$, $(2,8,4),(7,11,2),(1,0,2),(3,11,0),(5,11,1),(1,10,2),(3,10,4),(0,12,2),(12$, $10,1),(14,10,1),(10,9,2),(9,7,3),(8,9,1),(10,9,4),(7,11,4),(9,11,4),(7,7$, $1),(7,7,3),(8,5,3),(11,12,1),(13,12,1),(14,0,0),(6,9,1),(3,11,2),(6,9,3)$, $(4,8,4),(6,5,0),(2,4,3),(1,0,0),(12,1,1),(1,6,1),(5,7,2),(2,8,2),(4,9,2)$, $(1,6,3),(3,6,4),(4,4,2),(6,5,2),(12,14,1),(11,12,3),(13,12,3),(12,5,4),(4$, $5,0),(5,7,0),(2,9,0),(4,9,0),(3,6,2),(11,7,4),(11,7,2),(12,6,0),(11,8,0)$, $(14,6,1),(0,8,1),(8,9,3),(9,3,3),(11,3,3),(4,4,4),(6,4,4),(3,7,0),(13,8$, $0),(13,8,2),(14,6,3),(0,8,3),(12,10,3),(14,10,3),(3,2,3),(5,3,0),(7,3,0)$, $(10,1,1),(5,2,4),(10,14,3),(8,13,4),(1,2,0),(14,2,0),(13,4,0),(0,4,1),(2$, $4,1),(0,4,3),(5,0,1),(14,14,2),(12,14,3),(0,12,4),(6,13,2),(5,0,3),(5,11$, $3),(6,13,4),(9,3,1),(11,3,1),(8,5,1),(9,7,1),(10,5,2),(12,5,2),(10,5,4)$, $(5,6,4),(9,12,0),(10,14,1),(8,13,2),(3,0,3),(4,13,3),(0,13,0),(2,13,0),(4$, $13,1),(5,2,2),(7,2,2),(9,1,3),(7,0,4),(7,2,4),(3,0,1),(3,2,1),(14,1,2)$, $(12,1,3),(1,1,4),(14,1,4),(13,3,4),(8,1,0),(8,14,0),(7,0,2),(2,13,2),(2$, $12,4),(1,14,4),(14,14,4)$

\section{References}

[1] N. Alon, On the capacity of digraphs, European J. Combinatorics 19 (1998) $1-5$.

[2] N. Alon, A. Orlitsky, Repeated communication and Ramsey graphs, IEEE Trans. Inform. Theory 41 (1995) 1276-1289.

[3] J. J. Ashley, P. H. Siegel, A note on the Shannon capacity of run-lengthlimited codes, IEEE Trans. Inform. Theory 33 (1987) 601-605.

[4] L. D. Baumert, R. J. McEliece, E. Rodemich, H. C. Rumsey Jr., R. Stanley, and H. Taylor, A combinatorial packing problem, In: Proc. SIAM-AMS Sympos. Appl. Math., New York, 1970, Computers in Algebra and Number Theory, G. Birkhoff and M. Hall Jr. (Eds.), Amer. Math. Soc., Providence, 1971, pp. 97-108.

[5] T. Bohman, A limit theorem for the Shannon capacities of odd cycles I, Proc. Amer. Math. Soc. 131 (2003) 3559-3569.

[6] T. Bohman, R. Holzman, V. Natarajan, On the independence numbers of the cubes of odd cycles, Electron. J. Combin. 20(3) (2013) \#P10.

[7] I. M. Bomze, M. Budinich, P. M. Pardalos, and M. Pelillo, The maximum clique problem, In: Handbook of Combinatorial Optimization, Supplement 
Vol. A, D. Z. Du and P. M. Pardalos (Eds.), Kluwer, Dordrecht, 1999, pp. $1-74$.

[8] M. Braun, P. R. J. Östergård, A. Wassermann, New lower bounds for binary constant dimension subspace codes, in preparation.

[9] A. E. Brouwer, A. Schrijver, Uniform hypergraphs, In: Packing and Covering in Combinatorics, A. Schrijver (Ed.), Math. Centre Tracts No. 106, Mathematisch Centrum, Amsterdam, 1979, pp. 39-73.

[10] U. Feige, Randomized graph products, chromatic numbers, and the Lovász $\vartheta$-function, Proc. 27th Annual ACM Symposium on the Theory of Computing, 1995, pp. 635-640.

[11] R. Frucht, On the groups of repeated graphs, Bull. Amer. Math. Soc. 55 (1949), 418-420.

[12] W. Haemers, An upper bound for the Shannon capacity of a graph, In: Algebraic Methods in Graph Theory, L. Lovász and V. T. Sós (Eds.), Colloq. Math. Soc. János Bolyai, Vol. 25, Szeged, Hungary, 1978, pp. 267-272.

[13] W. Haemers, On some problems of Lovász concerning the Shannon capacity of a graph, IEEE Trans. Inform. Theory 25 (1979) 231-232.

[14] R. Hammack, W. Imrich, S. Klavžar, Handbook of Product Graphs, Second Edition, Discrete Mathematics and Its Applications, CRC Press, Boca Raton, Florida, 2011.

[15] P. Kaski, P. R. J. Östergård, Classification Algorithms for Codes and Designs, Springer-Verlag, Berlin, 2006.

[16] O. H. Keller, Über die lückenlose Erfüllung des Raumes mit Würfeln, J. Reine Angew. Math. 163 (1930), 231-248.

[17] D. E. Knuth, The sandwich theorem, Electron. J. Combin. 1, 1994, \#A1.

[18] J. Körner, A. Orlitsky, Zero-error information theory, IEEE Trans. Inform. Theory 44 (1998) 2207-2229.

[19] L. Lovász, On the Shannon capacity of a graph, IEEE Trans. Inform. Theory $25(1979) 1-7$.

[20] K. A. Mathew, P. R. J. Östergård, A. Popa, On the Shannon capacity of triangular graphs, Electron. J. Combin., 20(2) (2013) \#P27.

[21] R. Montemanni and D. H. Smith, Heuristic algorithms for constructing binary constant weight codes, IEEE Trans. Inform. Theory 55 (2009) 46514656.

[22] S. Niskanen, P. R. J. Östergård, Cliquer User's Guide (version 1.0), Technical report T48, Commun. Lab., Helsinki Univ. Technol., Espoo, 2003. 
[23] P. R. J. Östergård, Partial neighborhood search for the maximum weight clique problem, in preparation.

[24] C. E. Shannon, The zero-error capacity of a noisy channel, IRE Trans. Inform. Theory 2 (1956) 8-19.

[25] A. Vesel, J. Žerovnik, Improved lower bound on the Shannon capacity of $C_{7}$, Inform. Process. Lett. 81 (2002) 277-282.

[26] M. Szegedy, A note on the $\Theta$ number of Lovász and the generalized Delsarte bound, Proc. 35h Annual Symposium on Foundations of Computer Science, IEEE Computer Society Press, 1994, pp. 36-41. 